VOL. 52 (1995) [487-498]

\title{
GENERIC DIFFERENTIABILITY OF LOCALLY LIPSCHITZ FUNCTIONS ON PRODUCT SPACES
}

\author{
J.R. Giles
}

\begin{abstract}
Although it is known that locally Lipschitz functions are densely differentiable on certain classes of Banach spaces, it is a minimality condition on the subdifferential mapping of the function which enables us to guarantee that the set of points of differentiability is a residual set. We characterise such minimality by a quasi continuity property of the Dini derivatives of the function and derive sufficiency conditions for the generic differentiability of locally Lipschitz functions on a product space.
\end{abstract}

\section{INTRODUCTION}

A real valued function $\psi$ on an open subset $A$ of a normed linear space $X$ is locally Lipschitz if for each $x_{0} \in A$ there exists a $K_{0}>0$ and $\delta_{0}>0$ such that

$$
|\psi(x)-\psi(y)| \leqslant K_{0}\|x-y\| \text { for all } x, y \in B\left(x_{0} ; \delta_{0}\right) .
$$

The function $\psi$ is Gateaux differentiable at $x \in A$ in the direction $y \in X$ if

$$
\psi^{\prime}(x)(y) \equiv \lim _{\lambda \rightarrow 0} \frac{\psi(x+\lambda y)-\psi(x)}{\lambda}
$$

exists and is Gâteaux differentiable at $x \in A$ if it is Gâteaux differentiable at $x$ in all directions $y \in X$ and $\psi^{\prime}(x)$ is a continuous linear functional on $X$. The function $\psi$ is Fréchet differentiable at $x \in A$ if it is Gâteaux differentiable at $x$ and the limit is approached uniformly for all $y \in S(X)$. A Banach space $X$ is said to be smoothable if there exists an equivalent norm on $X$ which is Gâteaux differentiable everywhere except at the origin. A Banach space $X$ is an Asplund space if every continuous convex function on an open convex subset of $X$ is Fréchet differentiable on a residual subset of its domain.

The determination of differentiability properties of locally Lipschitz functions is particularly important for applications in optimisation. The differentiability of a locally

Received 28th February, 1995

Copyright Clearance Centre, Inc. Serial-fee code: 0004-9729/95 \$A2.00+0.00. 
Lipschitz function $\psi$ on an open subset $A$ of a normed linear space $X$ is studied using the Clarke directional derivative

$$
\psi^{0}(x)(y) \equiv \limsup _{\substack{z \rightarrow x \\ \lambda \rightarrow 0+}} \frac{\psi(z+\lambda y)-\psi(z)}{\lambda}
$$

at each $x \in A$ in the direction $y \in X$ and $\psi^{0}(x)(y)$ is a continuous sublinear functional in $y$. The Clarke subdifferential

$$
\partial \psi^{0}(x) \equiv\left\{f \in X^{*}: f(y) \leqslant \psi^{0}(x)(y) \text { for all } y \in X\right\}
$$

at each $x \in A$, is a non-empty weak* compact convex set.

The key result generalising the classical Rademacher Theorem from Euclidean to Banach spaces was given by David Preiss, [14].

Preiss' TheOREM. A locally Lipschitz function $\psi$ on an open subset $A$ of a smoothable (Asplund) space is Gâteaux (Fréchet) differentiable on a dense subset $D$ of $A$ and the Clarke subdifferential is generated by the Gateaux (Fréchet) derivatives; that is, given $x \in A$

$$
\partial \psi^{0}(x)=\bigcap_{r>0} \overline{c o}^{*}\left\{\psi^{\prime}(z): z \in B(x ; r) \cap D\right\}
$$

However, the set of points of differentiability need not be a residual subset of the domain and this can inhibit our analysis.

A set-valued mapping $\Phi$ from a topological space $A$ into subsets of a linear topological space $X$ is upper semi-continuous at $a \in A$ if given an open subset $W$ of $X$ such that $\Phi(a) \subseteq W$ there exists an open neighbourhood $U$ of $a$ such that $\Phi(U) \subseteq W$. When $\Phi$ is upper semi-continuous on $A$ and $\Phi(a)$ is convex and compact for each $a \in A$ we call $\Phi$ a cusco on $A$. We say that $\Phi$ is a minimal cusco on $A$ if its graph does not contain the graph of any other cusco with the same domain.

For a locally Lipschitz function $\psi$ on an open subset $A$ of a normed linear space $X$, the Clarke subdifferential mapping $x \mapsto \partial \psi^{0}(x)$ is a weak* cusco on $A$ but is not in general a minimal weak* cusco.

A locally Lipschitz function $\psi$ on an open subset $A$ of a normed linear space $X$ is said to be strictly differentiable at $x \in A$ in the direction $y \in X$ if

$$
\lim _{\substack{x \rightarrow x \\ \lambda \rightarrow 0+}} \frac{\psi(z+\lambda y)-\psi(z)}{\lambda}
$$

exists and is said to be strictly differentiable at $x$ if it is strictly differentiable at $x$ in all directions $y \in X$. Further, $\psi$ is said to be uniformly strictly differentiable at $x$ if this 
limit is approached uniformly for all $y \in S(X)$. Obviously, if $\psi$ is strictly differentiable at $x \in A$ then $\psi$ is Gâteaux differentiable at $x$. Further, if $\psi$ is uniformly strictly differentiable at $x \in A$ then $\psi$ is Fréchet differentiable at $x$.

Clearly, $\psi$ is strictly differentiable at $x \in A$ if and only if $\partial \psi^{0}(x)$ is singleton. But also, $\psi$ is uniformly strictly differentiable at $x \in A$ if and only if $\partial \psi^{0}(x)$ is singleton and the subdifferential mapping $x \mapsto \partial \psi^{0}(x)$ is norm upper semi-continuous at $x$, [5, p.374]. With certain minimal weak* cuscos we can associate significant residual subsets of the domain.

Proposition 1.1. Consider a minimal weak* cusco $\Phi$ from a Baire space $A$ into subsets of the dual $X^{*}$ of a Banach space $X$.

(i) If $X$ is smoothable then $\Phi$ is single-valued on a residual subset of $A$, [15].

(ii) If $X$ is Asplund then $\Phi$ is single-valued and norm upper semi-continuous at the points of a residual subset of $A,[12$, p.106].

The implications for differentiability of locally Lipschitz functions are immediate.

Corollary 1.2. A locally Lipschitz function $\psi$ on an open subset $A$ of a smoothable (Asplund) space $X$ is strictly (uniformly strictly) differentiable on a residual subset of $A$ if the subdifferential mapping $x \mapsto \partial \psi^{0}(x)$ on $A$ is minimal.

To establish this minimality for the subdifferential mapping can be a problem so there is considerable value in determining properties sufficient to guarantee it. Some work has already been done in this area, $[\mathbf{1}, \mathbf{2}, \mathbf{3}]$ and more recently [11].

Here we give a characterisation of minimality for the subdifferential mapping using quasi continuity and provide two sufficiency conditions for minimality on a product space. This in turn enables us to deduce sufficiency conditions for the generic differentiability of locally Lipschitz functions on a product space.

\section{A CHARACTERISATION OF MINIMAL SUBDIFFERENTIAL MAPPINGS}

The minimality of a cusco has the following useful characterisation, [8, p.252].

Lemma 2.1. A cusco $\Phi$ from a topological space $A$ into subsets of a separated locally convex $X$ is a minimal cusco if and only if for any open set $U$ in $A$ and open half-space $W$ in $X$ where $\Phi(U) \cap W \neq \emptyset$, there exists a non-empty open set $V \subseteq U$ such that $\Phi(V) \subseteq W$.

Proof: Suppose that $\Phi$ is a minimal cusco on $A$ and for an open set $U \subseteq A$ and open half-space $W$ we have $\Phi(U) \cap W \neq \emptyset$. If there exists an $a \in U$ such that $\Phi(a) \subseteq W$ then by the upper semi-continuity of $\Phi$ there exists a non-empty open neighbourhood $V$ of $a$ such that $\Phi(V) \subseteq W$. If not, then $\Phi(a) \cap C(W) \neq \emptyset$ for every 
$a \in U$. Consider the set-valued mapping $\Psi$ from $A$ into subsets of $X$ where

$$
\Psi(a)= \begin{cases}\Phi(a) \cap C(W) & \text { for } a \in U \\ \Phi(a) & \text { for } a \notin U .\end{cases}
$$

Then $\Psi$ is a cusco on $A$ whose graph is contained in that of $\Phi$. But this contradicts the minimality of $\Phi$

Conversely, suppose that $\Phi$ is a cusco which is not minimal. Then there exists a cusco $\Psi$ whose graph is contained in that of $\Phi$ but for some $a_{0} \in A$ there exists an $x_{0} \in \Phi\left(a_{0}\right) \backslash \Psi\left(a_{0}\right)$. Since $\Psi\left(a_{0}\right)$ is convex and compact there exist disjoint open half spaces $W_{1}$ and $W_{2}$ such that $\Psi\left(a_{0}\right) \subseteq W_{1}$, and $x_{0} \in W_{2}$. Since $\Psi$ is upper semicontinuous at $a_{0}$ there exists an open neighbourhood $U$ of $a_{0}$ such that $\Psi(U) \subseteq W_{1}$. But then $\Phi(U) \cap W_{2} \neq \emptyset$ and $\Phi(a) \cap C\left(W_{2}\right) \neq \emptyset$ for all $a \in U$.

The minimality of a weak* cusco can be characterised by the minimality of associated cuscos into subsets of the real numbers, [11, Proposition 1.4].

Lemma 2.2. Consider a weak* cusco $\Phi$ from a topological space $A$ into subsets of $X^{*}$ the dual of a Banach space $X$. Then $\Phi$ is a minimal weak* cusco if and only if for each $x \in S(X)$ the set-valued mapping $T_{x}$ from $A$ into subsets of $\mathbb{R}$ where $a \mapsto T_{x}(a)=\widehat{x}(\Phi(a))$ is a minimal cusco.

Proof: Suppose that $\Phi$ is a minimal weak* cusco on $A$. Given $x \in S(X)$ it is easy to see that $T_{x}$ is a cusco; we show that $T_{x}$ is minimal. Given $\alpha \in \mathbb{R}$ and an open set $U$ in $A$ such that $T_{x}(U) \cap(\alpha, \infty) \neq \emptyset$ then for some $a \in U$ and $f \in \Phi(a)$ we have $\widehat{x}(f)>\alpha$. Consider $W$ the open half-space, $W \equiv\left\{f \in X^{*}: f(x)>\alpha\right\}$. Now $\Phi(U) \cap W \neq \emptyset$. But since $\Phi$ is a minimal weak* cusco, from Lemma 2.1 there exists a non-empty open set $V \subseteq U$ such that $\Phi(V) \subseteq W$. That is, $\widehat{x}(\Phi(a))>\alpha$ for all $a \in V$ which implies that $T_{x}(V) \subseteq(\alpha, \infty)$. A similar argument applies for subsets of $\mathbb{R}$ of the form $(-\infty, \alpha)$ and we conclude from Lemma 2.1 that $T_{x}$ is a minimal cusco on $A$.

Conversely, suppose that $\Phi$ is not a minimal weak* cusco on $A$. Then there exists a weak* cusco $\Psi$ on $A$ whose graph is strictly contained in that of $\Phi$. So there exists an $a_{0} \in A$ such that $\Psi\left(a_{0}\right) \varsubsetneqq \Phi\left(a_{0}\right)$ and an $x_{0} \in S(X)$ such that max $\widehat{x}_{0}\left(\Phi\left(a_{0}\right)\right)>\max \widehat{x}_{0}\left(\Psi\left(a_{0}\right)\right)$. Now consider the two set-valued mappings $T_{x_{0}}$ and $\mathcal{S}_{x_{0}}$ from $A$ into subsets of $\mathbb{R}$ where $a \mapsto T_{x_{0}}(a)=\widehat{x}_{0}(\Phi(a))$ and $a \mapsto S_{x_{0}}(a)=\widehat{x}_{0}(\Psi(a))$. Clearly, $S_{x_{0}}(a) \subseteq T_{x_{0}}(a)$ for all $a \in A$. However, $\max S_{x_{0}}\left(a_{0}\right)=\max \widehat{x}_{0}\left(\Psi\left(a_{0}\right)\right)<$ $\max \widehat{x}_{0}\left(\Phi\left(a_{0}\right)\right)=\max T_{x_{0}}\left(a_{0}\right)$ so $S_{x_{0}}\left(a_{0}\right) \neq T_{x_{0}}\left(a_{0}\right)$ and we conclude that $T_{x_{0}}$ is not a minimal cusco on $A$.

For a locally Lipschitz function $\psi$ on an open subset $A$ of a normed linear space $X$, the upper Dini derivative of $\psi$ at $x \in A$ in the direction $y \in X$ is

$$
\psi^{+}(x)(y) \equiv \limsup _{\lambda \rightarrow 0+} \frac{\psi(x+\lambda y)-\psi(x)}{\lambda}
$$


and the lower Dini derivative of $\psi$ at $x \in A$ in the direction $y \in X$ is

$$
\psi^{-}(x)(y) \equiv \liminf _{\lambda \rightarrow 0+} \frac{\psi(x+\lambda y)-\psi(x)}{\lambda} .
$$

An equivalent formulation for the subdifferential of $\psi$ at $x \in A$ is

$$
\partial \psi^{0}(x) \equiv\left\{f \in X^{*}:-(-\psi)^{0}(x)(y) \leqslant f(y) \leqslant \psi^{0}(x)(y) \text { for all } y \in X\right\}
$$

and we note that

$$
-(-\psi)^{0}(x)(y)=\liminf _{\substack{x \rightarrow x \\ \lambda \rightarrow 0+}} \frac{\psi(z+\lambda y)-\psi(z)}{\lambda} .
$$

It is convenient to express the Clarke directional derivatives in terms of the directional and Dini derivatives, $[7$, p.837].

Lemma 2.3. Consider a locally Lipschitz function $\psi$ on an open subset $A$ of a normed linear space $X$. Given $y \in X$ and $x \in A$,

$$
\begin{aligned}
\psi^{0}(x)(y) & =\limsup _{\substack{z \rightarrow x \\
z \in D_{y}}} \psi^{\prime}(z)(y)=\limsup _{z \rightarrow x} \psi^{+}(z)(y) \\
-(-\psi)^{0}(x)(y) & =\liminf _{\substack{z \rightarrow x \\
z \in D_{y}}} \psi^{\prime}(z)(y)=\liminf _{z \rightarrow x} \psi^{-}(z)(y)
\end{aligned}
$$

where $D_{y}$ is the set of points in $A$ where $\psi$ is Gâteaux differentiable in the direction $y$.

ProOF: Clearly, $\psi^{0}(x)(y) \geqslant \underset{z \rightarrow x}{\limsup } \psi^{+}(z)(y) \geqslant \underset{\substack{x \rightarrow x \\ z \in D_{y}}}{\limsup } \psi^{\prime}(z)(y)$. But also, given $\varepsilon>0$, in any neighbourhood of $x$ there exists a $z_{0} \in A$ and $\lambda_{0}>0$ such that $z_{0}+\lambda_{0} y \in A$ and

$$
\frac{\psi\left(z_{0}+\lambda_{0} y\right)-\psi\left(z_{0}\right)}{\lambda_{0}}>\psi^{0}(x)(y)-\varepsilon .
$$

Consider $\psi$ restricted to the interval $\left[z_{0}, z_{0}+\lambda_{0} y\right]$. Since $\psi$ is locally Lipschitz it follows from Lebesgue's Differentiation Theorem that there exists a $0 \leqslant \lambda_{1} \leqslant \lambda_{0}$ such that

$$
\psi^{\prime}\left(z_{0}+\lambda_{1} y\right)(y) \geqslant \frac{\psi\left(z_{0}+\lambda_{0} y\right)-\psi\left(z_{0}\right)}{\lambda_{0}} .
$$

So $\underset{z \rightarrow x}{\limsup } \psi^{+}(z)(y) \geqslant \limsup _{\substack{z \rightarrow x \\ z \in D_{y}}} \psi^{\prime}(z)(y) \geqslant \psi^{0}(x)(y)$ and our first result follows. Now for all $x \in A$ and $y \in X,-(-\psi)^{0}(x)(y)=-\psi^{0}(x)(-y)$ and $\psi^{-}(x)(y)=$ $-(-\psi)^{+}(x)(y)$. So $-(-\psi)^{0}(x)(y)=-\underset{\substack{z \rightarrow x \\ z \in D_{y}}}{\limsup } \psi^{\prime}(z)(-y)=\liminf _{\substack{z \rightarrow D_{y} \\ z \in D_{y}}} \psi^{\prime}(z)(y)$. But also $-(-\psi)^{0}(x)(y)=-\limsup _{\substack{z \rightarrow x \\ z \in D_{y}}}(-\psi)^{\prime}(z)(y)=-\limsup _{z \rightarrow x}(-\psi)^{+}(z)(y)=\liminf _{z \rightarrow x} \psi^{-}(z)(y)$. 
From Preiss' Theorem we see that for a locally Lipschitz function on an open subset of a smoothable (Asplund) space the subdifferential is generated by the dense set of derivatives of the function and so in this case we have a tighter result.

Lemma 2.4. Consider a locally Lipschitz function $\psi$ on an open subset $A$ of a smoothable (Asplund) space $X$. Given $y \in X$ and $x \in A$,

$$
\begin{aligned}
\psi^{0}(x)(y) & =\underset{\substack{z \rightarrow x \\
z \in D}}{\limsup } \psi^{\prime}(z)(y)=\limsup _{z \rightarrow x} \psi^{+}(z)(y) \\
-(-\psi)^{0}(x)(y) & =\liminf _{\substack{z \rightarrow x \\
z \in D}} \psi^{\prime}(z)(y)=\liminf _{z \rightarrow x} \psi^{-}(z)(y)
\end{aligned}
$$

where $D$ is the set of points where $\psi$ is Gâteaux (Fréchet) differentiable on $A$.

Consider a real valued function $\phi$ on a topological space $A$. Now $\phi$ is said to be quasi upper semi-continuous at $a_{0} \in A$ if given $\varepsilon>0$ and an open neighbourhood $U$ of $a_{0}$, there exists a non-empty open set $V \subseteq U$ such that $\phi(a)<\phi\left(a_{0}\right)+\varepsilon$ for all $a \in V$, and is said to be quasi lower semi-continuous at $a_{0} \in A$ if $-\phi$ is quasi upper semi-continuous at $a_{0}$. The function $\phi$ is said to be quasi continuous at $a_{0} \in A$ if given $\varepsilon>0$ and an open neighbourhood $U$ of $a_{0}$, there exists a non-empty open set $V \subseteq U$ such that

$$
\phi\left(a_{0}\right)-\varepsilon<\phi(a)<\phi\left(a_{0}\right)+\varepsilon \text { for all } a \in V,
$$

If $\phi$ is quasi upper semi continuous on a Baire space $A$ then $\phi$ is continuous on a residual subset of $A,[4, \mathrm{p} .369]$.

We now present our characterisation for minimality of the Clarke subdifferential mapping in terms of quasi-continuity. The result is similar to that given in $[11$, Theorem 2.14].

Theorem 2.5. For a locally Lipschitz function $\psi$ on an open subset $A$ of a normed linear space $X$, the following are equivalent.

(i) the Clarke subdifferential mapping $x \mapsto \partial \psi^{0}(x)$ is a minimal weak ${ }^{*}$ cusco on $A$,

(ii) for each $y \in X, \psi^{+}(x)(y)$ is quasi upper semi-continuous on $A$,

(iii) for each $y \in X, \psi^{-}(x)(y)$ is quasi lower semi-continuous on $A$,

(iv) for each $y \in X, \psi^{\prime}(x)(y)$ is quasi upper semi-continuous on $D_{y}$,

(v) for each $y \in X, \psi^{\prime}(x)(y)$ is quasi lower semi-continuous on $D_{y}$,

where $D_{y}$ is the set of points in $A$ where $\psi$ is Gâteaux differentiable in the direction $\boldsymbol{y}$.

Proof: (i) $\Rightarrow$ (ii) Given $x \in A$ and $\varepsilon>0$ and any neighbourhood $U$ of $x$ there exists a non-empty open set $V \subseteq U$ such that

$$
\left[-(-\psi)^{0}(z)(y), \psi^{0}(z)(y)\right] \subseteq\left(-\infty,-(-\psi)^{0}(x)(y)+\varepsilon\right) \text { for all } z \in V .
$$


Then for each $z^{\prime} \in V$ there exists an open neighbourhood $V^{\prime}$ of $z^{\prime}$ where $V^{\prime} \subseteq V$ such that

$$
\psi^{+}(z)(y)<-(-\psi)^{0}(x)(y)+\varepsilon<\psi^{+}(x)(y)+\varepsilon \text { for all } z \in V^{\prime} ;
$$

that is, $\psi^{+}(x)(y)$ is quasi upper semi-continuous on $A$.

(i) $\Rightarrow$ (iii) Given $x \in A$ and $\varepsilon>0$ and any neighbourhood $U$ of $x$ there exists a non-empty open set $V \subseteq U$ such that

$$
\left[-(-\psi)^{0}(z)(y), \psi^{0}(z)(y)\right] \subseteq\left(\psi^{0}(x)(y)-\varepsilon, \infty\right) \text { for all } z \in V .
$$

Then as in (i) $\Rightarrow$ (ii) we deduce that $\psi^{-}(x)(y)$ is quasi lower semi-continuous on $A$.

(ii) $\Rightarrow$ (iv) and (iii) $\Rightarrow$ (v). It follows from Lebesgue's Differentiation Theorem that $D_{y}$ is dense in $A$ and so we have these results.

(iv) $\Leftrightarrow$ (v) Given $x \in D_{y}, \psi^{\prime}(x)(y)=-\psi^{\prime}(x)(-y)$. So $\psi^{\prime}(x)(-y)$ is quasi upper semi-continuous on $D_{y}$ if and only if $\psi^{\prime}(x)(y)$ is quasi lower semi-continuous on $D_{y}$.

(iv) $\Rightarrow$ (i) Given $x \in A$ and $\varepsilon>0$ and any neighbourhood $U$ of $x$, by Lemma 2.3 there exists an $x^{\prime} \in U \cap D_{y}$ such that

$$
\psi^{\prime}\left(x^{\prime}\right)(y)<-(-\psi)^{0}(x)(y)+\frac{\varepsilon}{2} .
$$

Since $\psi^{\prime}(x)(y)$ is quasi upper semi-continuous at $x^{\prime}$, there exists a non-empty open set $V \subseteq U$ such that

$$
\psi^{\prime}(z)(y)<\psi^{\prime}\left(x^{\prime}\right)(y)+\frac{\varepsilon}{2} \text { for all } z \in V \cap D_{y} .
$$

But then

$$
\psi^{0}(z)(y) \leqslant \psi^{\prime}\left(x^{\prime}\right)(y)+\frac{\varepsilon}{2}<-(-\psi)^{0}(x)(y)+\varepsilon \text { for all } z \in V
$$

So

$$
\left[-(-\psi)^{0}(z)(y), \psi^{0}(z)(y)\right] \subseteq\left(-\infty,-(-\psi)^{0}(x)(y)+\varepsilon\right) \text { for all } z \in V .
$$

Now $\psi^{0}(x)(-y)=(-\psi)^{0}(x)(y)$ and $-(-\psi)^{0}(x)(-y)=-\psi^{0}(x)(y)$. So applying our results to $-y \in X$ and $x \in A$ and neighbourhood $U$ of $x$ there exists a non-empty open set $V \subseteq U$ such that

$$
\left[-(-\psi)^{0}(z)(-y), \psi^{0}(z)(-y)\right] \subseteq\left(-\infty,-(-\psi)^{0}(x)(-y)+\varepsilon\right)
$$

that is,

$$
\left[-\psi^{0}(z)(y),(-\psi)^{0}(z)(y)\right] \subseteq\left(-\infty,-\psi^{0}(x)(y)+\varepsilon\right) \text { for all } z \in V
$$

So

$$
\left[-(-\psi)^{0}(z)(y), \psi^{0}(z)(y)\right] \subseteq\left(\psi^{0}(x)(y)-\varepsilon, \infty\right) \text { for all } z \in V
$$


We conclude that the Clarke subdifferential mapping $x \mapsto \partial \psi^{0}(x)$ is a minimal weak* cusco on $A$.

Using Lemma 2.4 we have a tighter result for a locally Lipschitz function on an open subset of a smoothable (Asplund) space.

Theorem 2.6. For a locally Lipschitz function $\psi$ on an open subset $A$ of a smoothable (Asplund) space $X$, the Clarke subdifferential mapping $x \mapsto \partial \psi^{0}(x)$ is a minimal weak* cusco on $A$ if and only if for each $y \in X, \psi^{\prime}(x)(y)$ is quasi upper semicontinuous on $D$, the set of points in $A$ where $\psi$ is Gâteaux (Fréchet) differentiable.

The proof in one direction follows from Theorem 2.5 (i) $\Rightarrow$ (iv). In the other direction it is similar to Theorem 2.5 (iv) $\Rightarrow$ (i) but using Lemma 2.4 .

A locally Lipschitz function $\psi$ on an open subset $A$ of a normed linear space $X$ is strictly differentiable at $x \in A$ in the direction $y \in X$ if and only if $\psi^{+}(x)(y)$ is continuous at $x\left[7\right.$, p.837]. Using the fact that, given $x \in A, \psi^{+}(x)(y)$ is continuous in $y,[6, p .207]$ and the generic continuity of quasi upper semi-continuous functions, we can make the following deduction.

Corollary 2.7. For a locally Lipschitz function $\psi$ on an open subset $A$ of a separable Banach space $X$, if the subdifferential mapping $x \mapsto \partial \psi^{0}(x)$ is minimal then $\psi$ is strictly differentiable on a residual subset of $A$.

We should note that such a result is not true for non-separable spaces. On $\ell_{\infty}$ the semi-norm $p$ defined for $x \equiv\left\{x_{1}, x_{2}, \ldots, x_{n}, \ldots\right\}$ by $p(x)=\lim \sup \left|x_{n}\right|$, has a minimal subdifferential mapping $x \mapsto \partial p(x)$, but $p$ is nowhere Gâteaux differentiable, [12, p.13]. Further, the converse of Corollary 2.7 does not hold in general. Pompeiu [13], has given an example of a real valued differentiable function $\psi$ with a bounded non-negative derivative on an interval $(a, b)$ where the sets $\left\{x \in(a, b): \psi^{\prime}(x)=0\right\}$ and $\left\{x \in(a, b): \psi^{\prime}(x)>0\right\}$ are both dense in $(a, b)$. Clearly at each point of $\left\{x \in(a, b): \psi^{\prime}(x)>0\right\}, \psi^{\prime}$ is not quasi lower semi-continuous and so the subdifferential mapping $x \mapsto \partial \psi^{0}(x)$ is not minimal. However, since $\psi$ is differentiable on $(a, b), \psi$ is strictly differentiable on a residual subset of $(a, b),[6$, p.210].

At this stage it is worth noting that a real valued differentiable function $\psi$ on an interval $(a, b)$ with derivative $\psi^{\prime}$ continuous almost everywhere, has $\psi^{\prime}$ quasi continuous on $(a, b),[9, p .974]$, and so has a minimal subdifferential mapping $x \mapsto \partial \psi^{0}(x)$. On the other hand there exists a real-valued function $\psi$ on $(a, b)$ with bounded derivative which is quasi continuous on $(a, b)$ but where the derivative is discontinuous on a set of positive measure, [9, p.975].

A locally Lipschitz function $\psi$ on an open subset $A$ of a normed linear space $X$ is said to be pseudo-regular at $x \in A$ in the direction $y \in X$ if $\psi^{+}(x)(y)=\psi^{0}(x)(y)$ and pseudo-regular at $x$ if it is pseudo-regular at $x$ in all directions $y \in X$. Since 
$\psi^{0}(x)(y)=\underset{z \rightarrow x}{\limsup } \psi^{+}(z)(y)$, it follows that $\psi$ is pseudo-regular at $x \in A$ in the direction $y \in X$ if and only if $\psi^{+}(x)(y)$ is upper semi-continuous at $x,[7, p .836]$. So we can make the following deduction, [11, Theorem 2.5].

Corollary 2.8. A locally Lipschitz function $\psi$ which is pseudo-regular on an open subset $A$ of a normed linear space $X$, has a minimal subdifferential mapping $x \mapsto \partial \psi^{0}(x)$ on $A$.

\section{MiNIMAL SUBDIFFERENTIAL MAPPINGS ON PRODUCT SPACES}

Given topological spaces $X, Y$ and $Z$ and a function $\theta$ from $X \times Y$ into $Z$, we define for $p \in X$, the function $\theta_{p}$ from $Y$ into $Z$ where

$$
\theta_{p}(y)=\theta(p, y)
$$

and for $q \in Y$, the function $\theta_{q}$ from $X$ into $Z$ where

$$
\theta_{q}(x)=\theta(x, q)
$$

The following lemma relates separate and joint quasi continuity modelled on the proof of a similar result, $[10$, p.39].

LEMma 3.1. Consider a real valued function $\theta$ on $X \times Y$ where $X$ is a Baire space and $Y$ is second countable. If $\theta_{x}$ is quasi upper semi-continuous on $Y$ for all $x \in X$ and $\theta_{y}$ is both quasi upper and quasi lower semi-continuous on $X$ for all $y \in Y$ then $\theta$ is quasi upper semi-continuous on $X \times Y$.

Proof: Suppose that $\theta$ is not quasi upper semi-continuous at $(p, q) \in X \times Y$. Then there is an $r>0$ and a neighbourhood $U \times V$ of $(p, q)$ such that in every non-empty open subset of $U \times V$ there exists an $(x, y)$ such that

$$
\theta(x, y) \geqslant \theta(p, q)+r
$$

Since $\theta_{q}$ is quasi upper semi-continuous at $p$, there exists a non-empty open set $E \subseteq U$ such that

$$
\theta(x, q)<\theta(p, q)+\frac{r}{3} \text { for all } x \in E .
$$

Consider $\mathcal{V}$ a countable base for $Y$ and $\left\{V_{n}: n \in \mathbb{N}\right\}$ those elements from $\mathcal{V}$ contained in $V$. For each $n \in \mathbb{N}$, write

$$
A_{n} \equiv\left\{x \in E: \theta(x, y)<\theta(x, q)+\frac{r}{3} \text { for all } y \in V_{n}\right\}
$$


Consider $x \in E$. Since $\theta_{x}$ is quasi upper semi-continuous at $q$ there exists a nonempty open set $F \subseteq V$ such that $\theta(x, y)<\theta(x, q)+r / 3$ for all $y \in F$. But there exists $k \in \mathbb{N}$ such that $V_{k} \subseteq F$. So $x \in A_{k}$ and $E=\bigcup_{1}^{\infty} A_{n}$.

Consider $E^{\prime}$ a non-empty open subset of $E$ and $n \in \mathbb{N}$. Then $E^{\prime} \times V_{n} \subseteq U \times V_{n}$ and there is a $\left(x^{\prime}, y^{\prime}\right) \in E^{\prime} \times V_{n}$ such that $\theta\left(x^{\prime}, y^{\prime}\right) \geqslant \theta(p, q)+r$. Since $\theta_{y^{\prime}}$ is quasi lower semi-continuous at $x^{\prime}$, there exists a non-empty open set $E^{\prime \prime} \subseteq E^{\prime}$ such that

$$
\theta\left(x, y^{\prime}\right)>\theta\left(x^{\prime}, y^{\prime}\right)-\frac{r}{3} \text { for all } x \in E^{\prime \prime}
$$

For $x \in E^{\prime \prime}$,

$$
\theta\left(x, y^{\prime}\right)>\theta\left(x^{\prime}, y^{\prime}\right)-\frac{r}{3} \geqslant \theta(p, q)+\frac{2 r}{3}>\theta(x, q)+\frac{r}{3}
$$

But since $y^{\prime} \in V_{n}$ then $x \notin A_{n}$ and so $E^{\prime \prime} \cap A_{n}=\emptyset$. Therefore, $A_{n}$ is nowhere dense and $E$ is of first Baire category. This contradicts the fact that $X$ is a Baire space.

This Lemma with Theorem 2.5 gives an improved sufficiency theorem for minimal subdifferential mappings of locally Lipschitz functions on certain product spaces.

Theorem 3.2. Consider a locally Lipschitz function $\psi$ on a product space $X \times$ $Y$ where $X$ and $Y$ are Banach spaces and $Y$ is separable. The subdifferential mapping $(x, y) \mapsto \partial \psi^{0}(x, y)$ is minimal on $X \times Y$ if given $(u, v) \in X \times Y$, for each $p \in X$, $\psi^{+}(p, y)(u, v)$ is quasi upper semi-continuous on $Y$ and for each $q \in Y, \psi^{+}(x, q)(u, v)$ is both quasi upper and quasi lower semi-continuous on $X$.

From Theorem 3.2 and Proposition 1.1 we can deduce the following generic differentiability properties of locally Lipschitz functions on a product space.

Corollary 3.3. Consider a locally Lipschitz function $\psi$ a product space $X \times$ $Y$ where $X$ and $Y$ are Banach spaces and $Y$ is separable and $\psi$ satisfies the hypothesis of Theorem 3.2 .

(i) If $X$ is smoothable, then $\psi$ is strictly differentiable on a residual subset of $X \times Y$.

(ii) If $X$ is Asplund and $Y$ has separable dual, then $\psi$ is uniformly strictly differentiable on a residual subset of $X \times Y$.

\section{PROOF:}

(i) If $X$ is smoothable and $Y$ is separable, then $Y$ is smoothable and so $X \times Y$ is smoothable.

(ii) If $X$ is Asplund and $Y$ has separable dual, then closed separable subspaces of $X \times Y$ have separable duals and $X \times Y$ is Asplund, [12, p.32].

Our result now follows from Proposition 1.1 and Corollary 1.2. 
Theorem 3.2 provides a test for minimality for locally Lipschitz functions on a product space using the behaviour of associated functions on each of the component spaces. Our other theorem gives a similar result using the behaviour of derivatives in component directions.

Theorem 3.4. Consider a locally Lipschitz function $\psi$ on an open subset $A$ of a smoothable (Asplund) product space $X \times Y$ where $X$ and $Y$ are Banach spaces. The subdifferential mapping $(x, y) \mapsto \partial \psi^{0}(x, y)$ is minimal on $A$ if one of $\psi^{\prime}(x, y)(u, 0)$ and $\psi^{\prime}(x, y)(0, v)$ is upper semi-continuous on $D$ and the other is quasi upper semicontinuous on $D$ where $D$ is the set of points in $A$ where $\psi$ is Gâteaux (Fréchet) differentiable.

Proof: Given $(u, v) \in X \times Y$ and $(x, y) \in D$ then

$$
\psi^{\prime}(x, y)(u, v)=\psi^{\prime}(x, y)(u, 0)+\psi^{\prime}(x, y)(0, v)
$$

It follows that $\psi^{\prime}(x, y)(u, v)$ is quasi upper semi-continuous on $D$ and Theorem 2.6 gives our result.

In particular, $\psi$ satisfies the hypothesis of this theorem when $\psi$ is pseudo-regular on $X \times Y$, in directions $(u, 0)$ and $(0, v),[6, p .837]$. So Theorem 3.4 can be considered to be a generalisation of Corollary 2.8 .

From Theorem 3.4 and Proposition 1.1 we can deduce generic differentiability properties.

Corollary 3.5. A locally Lipschitz function $\psi$ on an open subset $A$ of a smoothable (Asplund) product space $X \times Y$ where $X$ and $Y$ are Banach spaces and $\psi$ satisfies the hypothesis of Theorem 3.4, has $\psi$ strictly (uniformly strictly) differentiable on a residual subset of $A$.

It is a classical result that a real valued locally Lipschitz function on Euclidean space with continuous partial derivatives at a point is strictly differentiable at the point. A proof of this follows from the more general local result.

Theorem 3.6. Consider a locally Lipschitz function $\psi$ on an open subset $A$ of a a product space $X \times Y$ where $X$ and $Y$ are normed linear spaces. If $\psi$ is strictly differentiable at $\left(x_{0}, y_{0}\right)$ in both directions $(u, 0)$ and $(0, v)$ then $\psi$ is strictly differentiable at $\left(x_{0}, y_{0}\right)$.

Proof: Consider $f \in \partial \psi^{0}\left(x_{0}, y_{0}\right)$. Since $\psi$ is strictly differentiable at $\left(x_{0}, y_{0}\right)$ in directions $(u, 0)$ and $(0, v)$ then

$$
f(u, 0)=\psi^{0}\left(x_{0}, y_{0}\right)(u, 0) \text { and } f(0, v)=\psi^{0}\left(x_{0}, y_{0}\right)(0, v) .
$$

So $f(u, v)=\psi^{0}\left(x_{0}, y_{0}\right)(u, v)$ and we conclude that $\partial \psi^{0}\left(x_{0}, y_{0}\right)$ is singleton. 


\section{REFERENCES}

[1] J.M. Borwein, 'Minimal cuscos and subgradients of Lipschitz functions', in Fixed point theory and its applications (Pitman Research Notes 252, 1991), pp. 57-81.

[2] J. Borwein, S. Fitzpatrick and P. Kenderov, 'Minimal convex uscos and monotone operators on small sets', Canad. J. Math. 43 (1991), 461-476.

[3] G. de Barra, S. Fitzpatrick and J.R. Giles, 'On generic differentiability of locally Lipschitz functions on Banach space', Proc. Centre Math. Anal. Austral. Nat. Univ. 20 (1988), $39-49$.

[4] J.R. Giles and M.O. Bartlett, 'Modified continuity and a generalisation of Michael's selection theorem, Set-Valued Anal. 1 (1993), 365-378.

[5] J.R. Giles and S. Sciffer, 'Continuity characterisations of differentiability of locally Lipschitz functions', Bull. Austral. Math. Soc. 41 (1990), 371-380.

[6] J.R. Giles and S. Sciffer, 'Locally Lipschitz functions are generically pseudo-regular on separable Banach spaces', Bull. Austral. Math. Soc. 47 (1993), 205-212.

[7] J.R. Giles and S. Sciffer, 'Generalising generic differentiability properties from convex to locally Lipschitz functions', J. Math. Anal. Appl. 188 (1994), 833-854.

[8] J.R. Giles and W.B. Moors, 'A continuity property related to Kuratowski's index of non-compactness, its relevance the the drop property and its implications for differentiability theory', J. Math. Anal. Appl. 178 (1993), 247-268.

[9] S. Marcus, 'Sur les fonctions dérivées, intégrables, au sens de Riemann et sur les dérivées partialles mixtes', Proc. Amer. Math. Soc. 9 (1958), 973-978.

[10] N.F.G. Martin, 'Quasi-continuous functions on product spaces', Duke Math. J. 28 (1961), $39-44$.

[11] W.B. Moors, 'A characterisation of minimal subgradients of locally Lipschitz functions', Set-Valued Anal. (to appear).

[12] R.R. Phelps, Convex functions, monotone operators and differentiability, Lecture Notes in Math. 1364 (Springer-Verlag, Berlin, Heidelberg, New York, 1993).

[13] D. Pompeiu, 'Sur les fonctions dérivées", Math. Ann. 63 (1907), 326-332.

[14] D. Preiss, 'Fréchet derivatives of Lipschitz functions', J. Funct. Anal. 91 (1990), 312-345.

[15] D. Preiss, R.R. Phelps and I. Namioka, 'Smooth Banach spaces, weak Asplund spaces and monotone or usco mappings', Israel J. Math. 72 (1990), 257-279.

Department of Mathematics

The University of Newcastle

New South Wales 2308

Australia 